\title{
Efficient Synthesis of 1,5-disubstituted-1H-tetrazoles by an Ugi-azide Process
}

\author{
Luis Edilberto Cárdenas-Galindo, ${ }^{1}$ Alejandro Islas-Jácome, ${ }^{1}$ Carlos Jesús Cortes-García, ${ }^{1}$ Laurent El \\ Kaim, ${ }^{2, *}$ and Rocío Gámez-Montaño ${ }^{1, *}$ \\ ${ }^{1}$ Departamento de Química, División de Ciencias Naturales y Exactas, Universidad de Guanajuato, Noria Alta S/N, C. P. \\ 36050, Guanajuato, Gto, México. Tel: +52-(473)-7320006, ext.8191 rociogm@ugto.mx \\ 2 UMR 7652, (Ecole Polytechnique/ENSTA/CNRS), Laboratoire Chimie et Procédés, Ecole Nationale Supérieure de \\ Techniques Avancées, 32 Bd Victor, 75015 Paris, France. Tel: +33-(145)-52553 laurent.elkaim@ensta.fr
}

Received January 22, 2013; Accepted May 28, 2013.

\begin{abstract}
A series of eighteen novel 1,5-disubstituted-1 $H$-tetrazoles has been prepared with moderate to good overall yields using an Ugi-azide reaction as key step under mild conditions. Tetrazoles are heterocyclic systems present in several synthetic products which have privileged biological activity. Therefore, in this paper we describe the synthesis of novel compounds containing the tetrazole moiety, which could present biological activity.

Keywords: Multicomponent reactions, Ugi-azide, 1,5 dipolar electrocyclization, tetrazole, azide, isocyanide.
\end{abstract}

\section{Introduction}

Tetrazoles are an important class of heterocycles, which are present in various compounds of great interest in medicinal chemistry because of their privileged biological activity. For example, losartan $\mathbf{1}$ is an angiotensin II receptor antagonist [1]. Tomelukast (L-171883, 2) [2], mimics the cystein-ylglycine terminus of growth hormone $\mathrm{LTD}_{4}$ and also functions as a potent anti-asthmatic drug [3] and BMS-317180 (3) is a potent oral agonist of the human growth hormone secretagogue (GHS) receptor [4], (Fig. 1).

Various methodologies for preparing compounds with a tetrazole ring system [5] have been developed, among the most important are those based on azides on cyanides [6] reactions, for example, Aldhoun et al described a short route based on [2, 3]-dipolar cycloadditions using TMSCN and several azides as starting materials to access a series of 1,5-disubstituted- $1 H$ tetrazoles with good to excellent overall yields [7]. Multicomponent reactions (MCR) have also been exploited to obtain
Resumen. Fue preparada una serie de dieciocho nuevos tetrazoles- $1 H$ 1,5-disubstituidos con rendimientos de moderados a buenos utilizando una reacción Ugi-azida como proceso clave bajo condiciones suaves. Los tetrazoles son sistemas heterocíclicos presentes en diversos productos sintéticos con actividad biológica privilegiada. En este trabajo describimos la síntesis de nuevos compuestos con el núcleo de tetrazol, los cuales podrían presentar actividad biológica.

Palabras clave: Reacciones de multicomponentes, Ugi-azida, electrociclización 1,5 dipolar, tetrazol, azida, isonitrilo.

compounds with a tetrazole ring system in short reactions time with good overall yields [8]. Hulme, who has been a pioneer in this field, reported in 2002 a rapid preparation of norstatine analogs, which include the tetrazole ring system based on a Passerini-type multicomponent reaction with $\mathrm{TMSN}_{3}$ and several isocyanides as starting materials in excellent overall yields [9]. Additionally, Kazemizadeh et al, recently published a novel methodology to prepare a series of 1,5-disubstituted-1H-tetrazoles which was based on the use of a three component reaction as key-step process using isocyanides, $\mathrm{TMSN}_{3}$ and several carbodiimides as starting materials [10]. Among multicomponent reactions, the Ugi-type MCR combined with subsequent postcondensation processes present several advantages over other methodologies for the 1,5-disubstituted- $1 H$-tetrazoles synthesis [11]. In this context, important efforts have been made by the Dömling's research group for the preparation of novel Ugitetrazoles of great interest in medicinal chemistry [12]. Also, Marcaccini reported the synthesis of a series of tetrazoyl isoindolinones via a tandem Ugi-4CR/amidation with moderate to

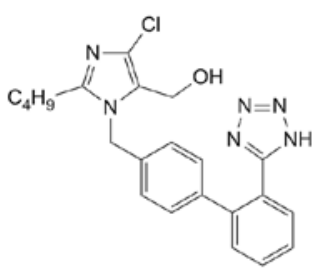

Losartan

(1)

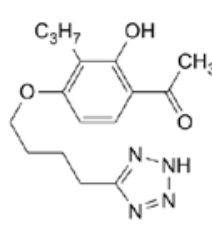

L-171883

(2, Tomelukast)

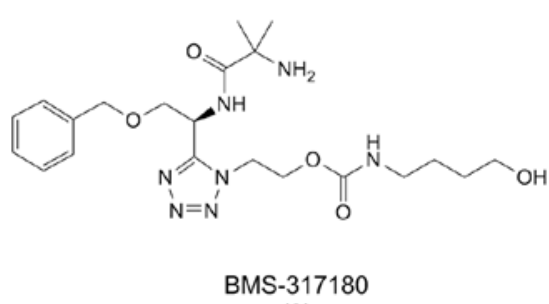

(3)

Fig. 1. Tetrazoles of great interest in medicinal chemistry. 
good overall yields [13]. More recently, Hulme et al published an elegant synthesis of a novel 1,5-disubstituted- $1 H$-tetrazoles series with moderate yields based on the Ugi-azide process [14].

The most important feature of the Ugi-3CR is the ease to construct structural diversity and to access to compound libraries by changing one of the starting materials [15]. In this context, series of different fused tetrazolopiperazines have been prepared with tosylate-substituted isocyanides[16], isocyanoesters [17], fluoroaryl isocyanides [18], or Schöllkopf isocyanides [19].

\section{Results and Discussion}

In this work, we describe a rapid preparation of a series of eighteen 1,5-disubstituted-1 $H$-tetrazoles (4a-r) with good to excellent overall yields (70-94\%) based on the use of a catalyst free optimized Ugi-azide process employing commercially available aryl-ethanamine derivatives $\mathbf{5}$, aldehydes $\mathbf{6}$, isocyanides 7 and $\mathrm{TMSN}_{3}(\mathbf{8})$ in $\mathrm{MeOH}$ under mild conditions (room temperature), (Scheme 1).

As depicted in Scheme 1, most of the compounds were obtained with really high yields, see for example $\mathbf{4 f}(91 \%)$, $4 \mathbf{i}(94 \%)$ and $4 \mathbf{p}(96 \%)$. Thus, yields are independent of the electronic and structural nature of substituents in the starting materials. As can be seen in the multicomponent process, aryl-ethanamines, aryl, alkyl aldehydes and isocyanides were used showing the generality of the methodology. In fact, no significant changes have been observed in the yields. Steric effects, which commonly take importance in the Ugi-3CR, had no influence on the yields of the corresponding 1,5-disubstituted-1 $H$-tetrazoles. Lower yields for the compounds $\mathbf{4 e}$ and $\mathbf{4 k}$ (which have the lowest steric factor) can be explained by the low boiling point of acetaldehyde, which hampers its experimental manipulation. Thus, the main contribution of our work is the operational simplicity of this multicomponent methodology to access to libraries of novel 1,5-disubstituted$1 H$-tetrazoles under mild conditions in relatively short reaction time. This latter is in comparison with other reports in which high temperatures, catalysts or strong acidic media are required [20].

We are currently exploiting a comprehensive study of the reactivity of other amines in this Ugi-azide process such as polysubstituted benzylamines and anilines. In this work, the resulting 1,5-disubstituted-1 $H$-tetrazoles (4a-r) are aryl-ethanamine derivatives with possible biological activity.

The reaction mechanism by which the synthesis of 1,5disubstituted- $1 H$-tetrazoles 4a-r occurs is depicted in Scheme 2. First, condensation occurs between amines $\mathbf{5}$ and aldehydes $\mathbf{6}$ to produce imines $\mathbf{9}$, which are converted into corresponding iminium ions 10 by hydrazoic acid. Then, isocyanide 7 undergoes an $\alpha$-nucleophilic addition to produce nitrilium ions 11, which are attacked by the azide anion to give intermediates 12, which carry out a 1,5 dipolar electrocyclization to afford the 1,5-disubstituted- $1 H$-tetrazoles series 4 [21].

\section{Conclusions}

We prepared a series of eighteen novel 1,5-disubstituted- $1 \mathrm{H}$ tetrazoles with good to excellent overall yields in one simple operational reaction step using mild conditions at room temperature. With these tetrazoles, we will study other combinations of multicomponent reactions with other miscellaneous post-condensations methodologies.

\section{Experimental Part}

Starting materials were obtained from Aldrich (México) and were used without further purification. IR Spectra: Perkin Elmer Spectrum 100FT-IR spectrometer, $v_{\max }$ in $\mathrm{cm}^{-1} .{ }^{1} \mathrm{H}$ and ${ }^{13} \mathrm{C}-\mathrm{NMR}$ Spectra: Bruker (400 and $100 \mathrm{MHz}$, resp.), Varian (300 and $75 \mathrm{MHz}$ resp.) and (200 and $50 \mathrm{MHz}$ resp.), in $\mathrm{CDCl}_{3}$; $\delta$ in ppm rel. to $\mathrm{Me}_{4} \mathrm{Si}$ as internal standard, $J$ in $\mathrm{Hz}$.

General method: Amine 5 (1.0 M) (1.0 equiv.) aldehyde 6 (1.0 equiv.), isocyanide 7 (1.0 equiv.) and azidotrimethylsilane (8) (1.0 equiv.) were disolved in $\mathrm{MeOH}$ in a round-bottom flask equipped with a magnetic stirrer bar. The resulting mixture was stirred for $6 \mathrm{~h}$ under an inert nitrogen atmosphere at room temperature. The solvent was evaporated under reduced pressure until dryness, then the reaction mixture was diluted with $\mathrm{CH}_{2} \mathrm{Cl}_{2}(20 \mathrm{~mL})$ and washed with brine $(10 \mathrm{~mL})$. The aqueous layer was extracted with $\mathrm{CH}_{2} \mathrm{Cl}_{2}(2 \times 10 \mathrm{~mL})$. The combined organic layer was dried over $\mathrm{NaSO}_{4}$. The resulting product was purified by column chromatography on silica gel using a mixture of Hex-AcOEt $(3: 1 \mathrm{~V} / \mathrm{V})$ as eluent to afford compounds (4a-r).

1-(1-cyclohexyl-1H-tetrazol-5-yl)-N-(3,4-dimethoxyphenethyl)-3-methylbutan-1-amine (4a). The general method was followed using homoveratrylamine (5a) $(300 \mathrm{mg}, 1.66$ $\mathrm{mmol}$ ), isovaleraldehyde (6a) (143 $\mathrm{mg}, 1.66 \mathrm{mmol})$, cyclohexylisocyanide (7a) $(181 \mathrm{mg}, 1.66 \mathrm{mmol})$, and azidotrimethylsilane (8) (191 mg, $1.66 \mathrm{mmol})$, producing $578 \mathrm{mg}(87 \%)$ of 4a as a yellow solid; $\mathrm{R}_{f}=0.61 \mathrm{Hex}-\mathrm{AcOEt}(3: 1 \mathrm{~V} / \mathrm{V})$; m.p. $62-63^{\circ} \mathrm{C}$; FT-IR (ATR) $v_{\max } 3345,1515,1260,1236,1094$, $1027 \mathrm{~cm}^{-1} ;{ }^{1} \mathrm{H}-\mathrm{NMR}\left(\mathrm{CDCl}_{3}, 400 \mathrm{MHz}\right): \delta 0.86(\mathrm{~d}, J=6.6$ $\mathrm{Hz}, 3 \mathrm{H}, \mathrm{CH}_{3}$ of $\left.i-\mathrm{Bu}\right), \delta 0.92\left(\mathrm{~d}, J=6.6 \mathrm{~Hz}, 3 \mathrm{H}, \mathrm{CH}_{3}\right.$ of $\left.i-\mathrm{Bu}\right)$, $1.25-1.40\left(\mathrm{~m}, 3 \mathrm{H}, \mathrm{CH}_{2}\right.$ of $\left.\mathrm{Cy}, \mathrm{CH}-23\right), 1.40-1.49(\mathrm{~m}, 2 \mathrm{H}$, $\mathrm{CH}_{2}$ of $\left.\mathrm{Cy}\right), 1.60-1.73\left(\mathrm{~m}, 2 \mathrm{H}, \mathrm{CH}_{2}-22\right), 1.75(\mathrm{~d}, J=8.4 \mathrm{~Hz}$, $1 \mathrm{H}, \mathrm{NH}-13), 1.83-2.05\left(\mathrm{~m}, 6 \mathrm{H}, \mathrm{CH}_{2}\right.$ of $\left.\mathrm{Cy}\right), 2.59-2.62(\mathrm{~m}, 1 \mathrm{H}$, $\left.\mathrm{CH}_{2}-14\right), 2.65-2.71\left(\mathrm{~m}, 3 \mathrm{H}, \mathrm{CH}_{2}-15, \mathrm{CH}_{2}-14\right), 3.85(\mathrm{~s}, 6 \mathrm{H}$, $\left.\mathrm{CH}_{3}-26,28\right), 4.21$ (t, $J=7.4 \mathrm{~Hz}, \mathrm{CH}-12$ ), 4.53 (tt, $J=11.5$, 3.7, Hz, 1H, CH-6), 6.65-6.68 (m, 2H, CH-20, 21), 6.78 (d, $J$ $=8.0 \mathrm{~Hz}, 1 \mathrm{H}, \mathrm{CH}-17) ;{ }^{13} \mathrm{C}-\mathrm{NMR}\left(\mathrm{CDCl}_{3}, 100 \mathrm{MHz}\right): \delta 22.9$, $22.9(24,29), 25.2(9), 25.3(23), 25.8,25.8,33.4,33.6$ (7, 8, 10, 11), 36.2 (15), 44.1 (22), 49.1 (14), 52.4 (12), 56.2, 56.3 $(26,28), 111.7,112.1,120.9$ (17, 20 ,21), 132.3 (16), 147.9, $149.3(18,19), 155.8(5)$.

$\mathrm{N}$-(3,4-dimethoxyphenethyl)-1-(1-(4-methoxybenzyl)-1Htetrazol-5-yl)-3-methylbutan-1-amine (4b). The general method was followed using homoveratrylamine (5a) $(400 \mathrm{mg}, 2.21$ $\mathrm{mmol}$ ), isovaleraldehyde (6a) (190 mg, $2.21 \mathrm{mmol})$, 4-me- 


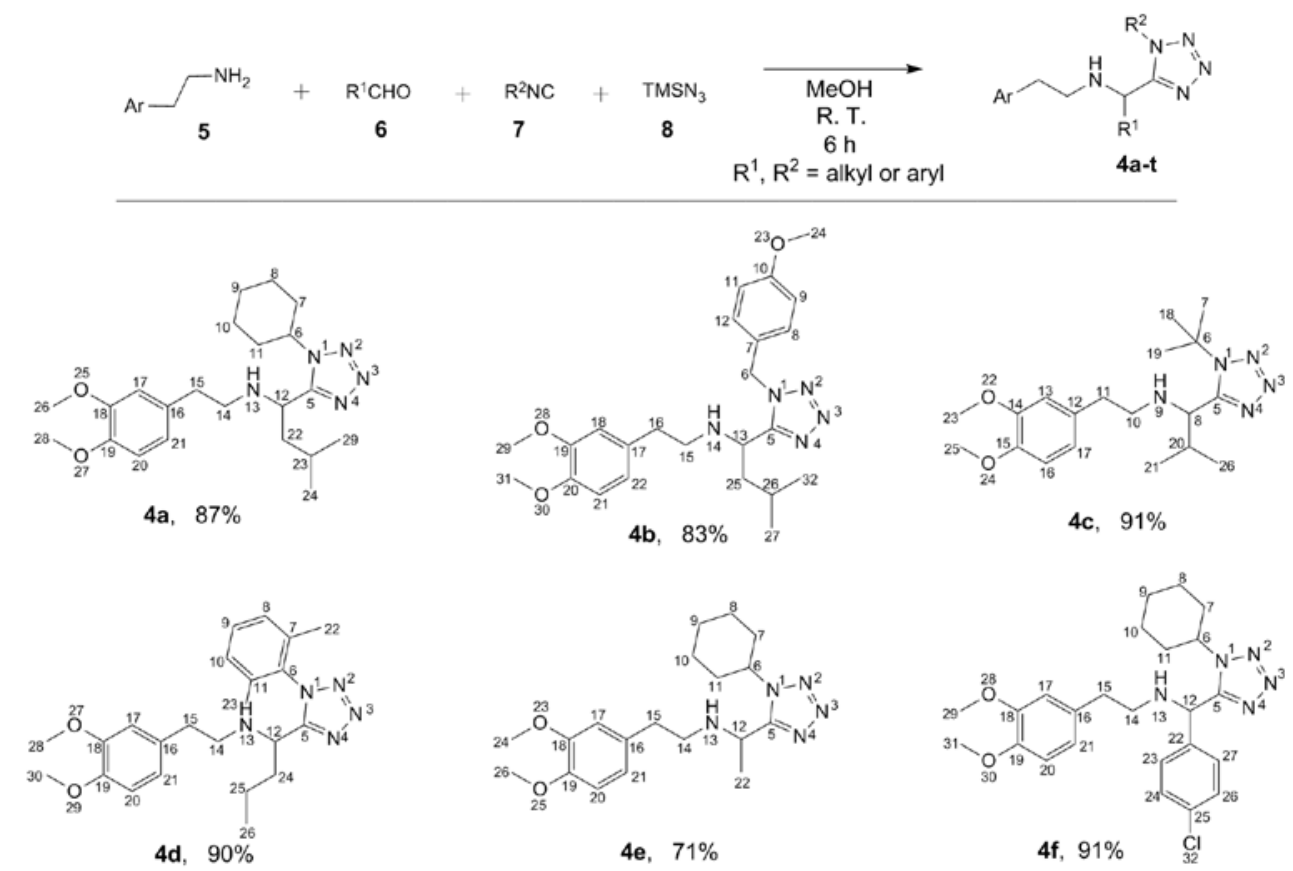

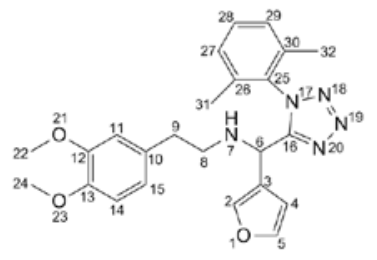

$4 \mathrm{~g}, 84 \%$

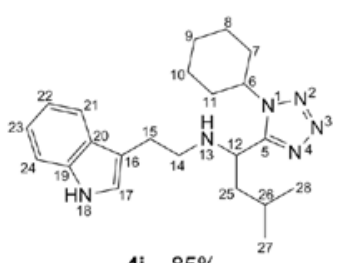

$4 \mathrm{j}, \quad 85 \%$

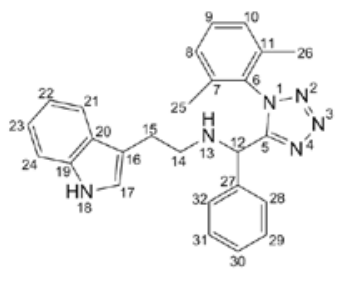

$4 m, \quad 78 \%$

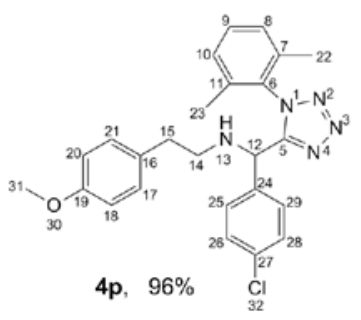

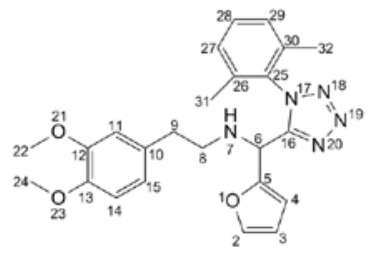

$4 h, 88 \%$

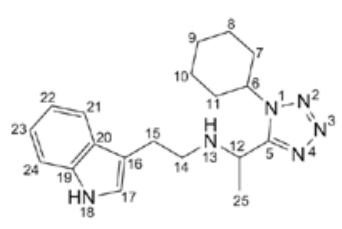

$4 \mathbf{k}, 74 \%$
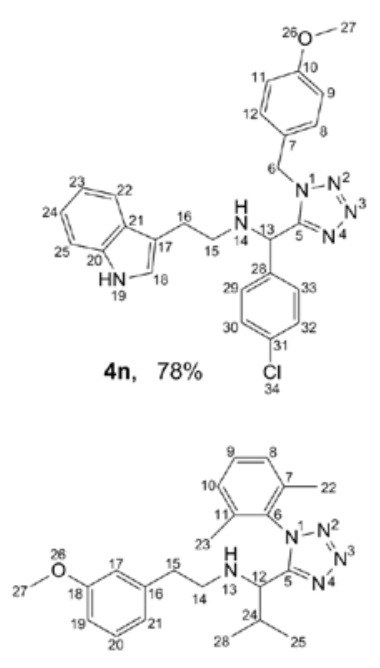

4 q. $89 \%$
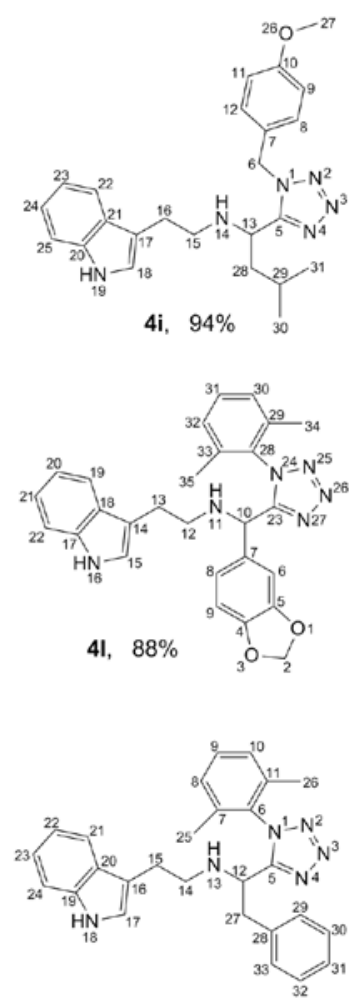

4o, $70 \%$

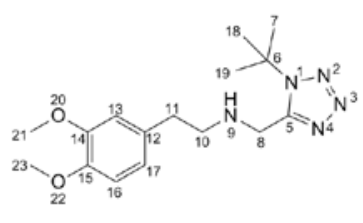

$4 r, \quad 80 \%$

Scheme 1. Synthesis of 1,5-disubstituted-1H-tetrazoles 4a-r. 


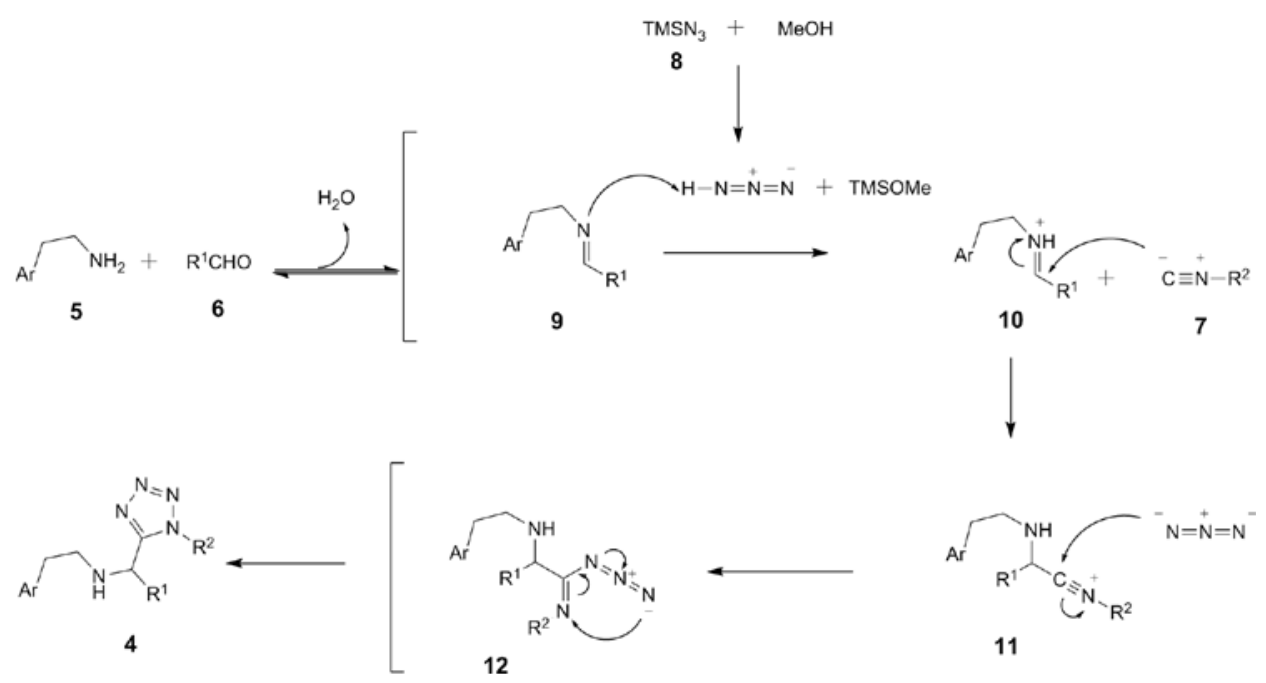

Scheme 2. Reaction mechanisms for the formation of 1,5-disubstituted-1H-tetrazoles 4a-r.

thoxy-benzylisocyanide (7b) (325 mg, $2.21 \mathrm{mmol})$, and azidotrimethylsilane (8) $(254 \mathrm{mg}, 2.21 \mathrm{mmol})$, producing $805 \mathrm{mg}$ $(83 \%)$ of $\mathbf{4 b}$ as a yellow oil; $\mathrm{R}_{f}=0.58 \mathrm{Hex}-\operatorname{AcOEt}(3: 1 \mathrm{~V} / \mathrm{V})$; FT-IR (ATR) $v_{\max } 3321,1513,1275,1258,1140,1027 \mathrm{~cm}^{-1}$; ${ }^{1} \mathrm{H}-\mathrm{NMR}\left(\mathrm{CDCl}_{3}, 400 \mathrm{MHz}\right): \delta 0.71\left(\mathrm{~d}, 3 \mathrm{H}, J=6.5 \mathrm{~Hz}, \mathrm{CH}_{3}\right.$ of $i-\mathrm{Bu}), 0.83\left(\mathrm{~d}, 3 \mathrm{H}, J=6.5 \mathrm{~Hz}, \mathrm{CH}_{3}\right.$ of $\left.i-\mathrm{Bu}\right), 1.30(\mathrm{~s}, 1 \mathrm{H}$, $\mathrm{NH}-14), 1.35$ (dt, $1 \mathrm{H}, J=13.7,6.7 \mathrm{~Hz}, \mathrm{CH}-26), 1.41-1.57$ (m, 2H, $\left.\mathrm{CH}_{2}-25\right), 2.51-2.63\left(\mathrm{~m}, 4 \mathrm{H}, \mathrm{CH}_{2}-15,16\right), 3.79$ (s, $3 \mathrm{H}$, $\left.\mathrm{CH}_{3}-24\right), 3.87$ (s, $\left.6 \mathrm{H}, \mathrm{CH}_{3}-29,31\right), 4.15(\mathrm{t}, 1 \mathrm{H}, J=7.5 \mathrm{~Hz}$, $\mathrm{CH}-13), 5.51$ (dd, $\left.2 \mathrm{H},{ }^{2} \mathrm{~J}=21.7,15.2 \mathrm{~Hz}, \mathrm{CH}_{2}-6\right), 6.63-6.66$ $(\mathrm{m}, 2 \mathrm{H}, \mathrm{CH}-21,22), 6.80(\mathrm{~d}, 1 \mathrm{H}, J=8.0 \mathrm{~Hz}, \mathrm{C} H-18), 6.86(\mathrm{~m}$, $2 \mathrm{H}, \mathrm{CH}-9,11), 7.14(\mathrm{~d}, 2 \mathrm{H}, J=8.7 \mathrm{~Hz}, \mathrm{CH}-8,12) ;{ }^{13} \mathrm{C}-\mathrm{NMR}$ $\left(\mathrm{CDCl}_{3}, 100 \mathrm{MHz}\right): \delta 22.6,22.8$ (27, 32), 25.1 (26), 36.1 (16), 43.5 (25), 49.0 (15), 50.9 (6), 52.3 (13), 55.7 (24), 56.3, 56.4 $(29,31), 111.7(18), 112.2(22), 114.7(9,11), 120.9(21), 126.5$ (7), $129.4(8,12), 132.4(17), 148.0,149.4(19,20), 156.8(5)$, $160.2(10)$.

1-(1-(tert-butyl)-1H-tetrazol-5-yl)-N-(3,4-dimethoxyphenethyl)-2-methylpropan-1-amine (4c). The general method was followed using homoveratrylamine (5a) $(250 \mathrm{mg}, 1.38$ $\mathrm{mmol})$, isobutyraldehyde (6b) (99 $\mathrm{mg}, 1.38 \mathrm{mmol}), t$-butyl isocyanide (7c) $(115 \mathrm{mg}, 1.38 \mathrm{mmol})$, and azidotrimethylsilane (8) $(159 \mathrm{mg}, 1.38 \mathrm{mmol})$, producing $454 \mathrm{mg}(91 \%)$ of $4 \mathbf{c}$ as a yellow oil; $\mathrm{R}_{f}=0.90 \mathrm{Hex}-\operatorname{AcOEt}(3: 1 \mathrm{~V} / \mathrm{V})$; FT-IR (ATR) $v_{\max }$ $3335,1514,1260,1235,1138,1027 \mathrm{~cm}^{-1}$; ${ }^{1} \mathrm{H}-\mathrm{NMR}\left(\mathrm{CDCl}_{3}\right.$, $200 \mathrm{MHz}$ ): $\delta 1.00\left(\mathrm{dd}, 6 \mathrm{H}, J=17.8,6.7 \mathrm{~Hz}, \mathrm{CH}_{3}-21,26\right), 1.72$ (s, 9H, $\left.\mathrm{CH}_{3}-7,18,19\right), 1.87$ (s, 1H, NH-9), 2.13 (dt, 1H, $J=$ 13.6, 7.0 Hz, $\mathrm{CH}-20), 2.65-2.70\left(\mathrm{~m}, 4 \mathrm{H}, \mathrm{CH}_{2}-10,11\right), 3.85$ (d, $\left.6 \mathrm{H}, J=1.3 \mathrm{~Hz}, \mathrm{CH}_{3}-23,25\right), 6.65-6.68(\mathrm{~m}, 2 \mathrm{H}, \mathrm{CH}-13$, 17), $6.77(\mathrm{~d}, 1 \mathrm{H}, J=8.7 \mathrm{~Hz}, \mathrm{CH}-16) ;{ }^{13} \mathrm{C}-\mathrm{NMR}\left(\mathrm{CDCl}_{3}, 50\right.$ MHz): $\delta$ 17.6, 20.5 (21, 26), $30.4(18,19), 32.8$ (20), 36.1 (11), 48.4 (10), 55.8, $55.9(23,25), 59.7$ (8), 61.3 (6), 11.2, 111.9 $(13,16), 120.5(17), 132.2(12), 147.4,148.8(14,15), 157.0$ (5).

$\mathrm{N}$-(3,4-dimethoxyphenethyl)-1-(1-(2,6-dimethylphenyl)1H-tetrazol-5-yl)butan-1-amine (4d). The general method was followed using homoveratrylamine (5a) (100 $\mathrm{mg}, 0.55 \mathrm{mmol}$ ), butyraldehyde (6c) (40 mg, $0.55 \mathrm{mmol}$ ), 2,6-dimethylphenyl isocyanide (7d) $(60 \mathrm{mg}, 0.55 \mathrm{mmol})$, and azidotrimethylsolane (8) $(64 \mathrm{mg}, 0.55 \mathrm{mmol})$, roducing $203 \mathrm{mg}(90 \%)$ of $4 \mathbf{d}$ as a yellow oil; $\mathrm{R}_{f}=0.68 \mathrm{Hex}-\operatorname{AcOEt}(3: 1 \mathrm{~V} / \mathrm{V})$; FT-IR (ATR) $v_{\max }$ 3323, 1514, 1260, 1235, 1139, $1027 \mathrm{~cm}^{-1}$; ${ }^{1} \mathrm{H}-\mathrm{NMR}\left(\mathrm{CDCl}_{3}\right.$, $300 \mathrm{MHz}): \delta 0.81\left(\mathrm{t}, 3 \mathrm{H}, J=7.3 \mathrm{~Hz}, \mathrm{CH}_{3}-26\right), 1.29(\mathrm{dq}, 2 \mathrm{H}, J=$ 14.9, $\left.7.5 \mathrm{~Hz}, \mathrm{CH}_{2}-25\right), 1.64-1.80$ (m, $\left.2 \mathrm{H}, \mathrm{CH}_{2}-24\right), 1.92$ (d, $6 \mathrm{H}$, $\left.J=4.5 \mathrm{~Hz}, \mathrm{CH}_{3}-22,23\right), 2.61-2.68\left(\mathrm{~m}, 2 \mathrm{H}, \mathrm{CH}_{2}-14\right), 2.68-2.84$ (m, 2H, CH $\left.\mathrm{CH}_{2}-15\right), 3.64$ (t, $\left.1 \mathrm{H}, J=6.5 \mathrm{~Hz}, \mathrm{CH}-12\right), 3.86$ (d, 6H, $J$ $\left.=1.8 \mathrm{~Hz}, \mathrm{CH}_{3}-28,30\right), 6.62-6.71(\mathrm{~m}, 2 \mathrm{H}, \mathrm{CH}-17,21), 6.74-6.82$ (m, 1H, CH-20), 7.15-7.26 (m, 2H, CH-8, 10), $7.38(\mathrm{t}, 1 \mathrm{H}, J=$ $7.6 \mathrm{~Hz}, \mathrm{CH}-9) ;{ }^{13} \mathrm{C}-\mathrm{NMR}\left(\mathrm{CDCl}_{3}, 75 \mathrm{MHz}\right): \delta 13.8(26), 17.5$, 17.6 (22, 23), 19.0 (25), 35.8 (24), 36.1 (15), 48.5 (14), 52.4 (12), 56.0, $56.0(28,30), 111.5(17), 112.0(20), 120.7$ (21), 129.0, 129.0 (8, 10), 130.9 (9), 132.0 (6), 132.2 (16), 135.7, $136.0(7,11), 147.6,149.0(18,19), 158.0(5)$.

1-(1-cyclohexyl-1H-tetrazol-5-yl)-N-(3,4-dimethoxyphenet hyl)ethanamine (4e). The general method was followed using homoveratrylamine (5a) $(500 \mathrm{mg}, 2.76 \mathrm{mmol})$, acetaldehyde (6d) (122 mg, $2.76 \mathrm{mmol}$ ), cyclohexylisocyanide (7a) (301 mg, $2.76 \mathrm{mmol})$, and azidotrimethylsilane (8) $(318 \mathrm{mg}, 2.76 \mathrm{mmol})$, producing $704 \mathrm{mg}(71 \%)$ of $\mathbf{4 e}$ as a yellow oil; $\mathrm{R}_{f}=0.42 \mathrm{Hex}-$ AcOEt (3:1 V/V); FT-IR (ATR) $v_{\text {max }} 3320,1514,1260,1235$, $1139,1026 \mathrm{~cm}^{-1} ;{ }^{1} \mathrm{H}-\mathrm{NMR}\left(\mathrm{CDCl}_{3}, 400 \mathrm{MHz}\right): \delta 1.22-1.39(\mathrm{~m}$, $4 \mathrm{H}, \mathrm{CH}$ of $\mathrm{Cy}), 1.47\left(\mathrm{~d}, 3 \mathrm{H}, J=6.8 \mathrm{~Hz}, \mathrm{CH}_{3}-22\right), 1.72(\mathrm{~d}, 1 \mathrm{H}$, $J=10.1 \mathrm{~Hz}, \mathrm{NH}-13), 1.84-2.00$ (m, $6 \mathrm{H}, \mathrm{CH}_{2}$ of $\left.\mathrm{Cy}\right), 2.62-2.70$ (m, 3H, $\left.\mathrm{CH}_{2}-15, \mathrm{CH}_{2}-14\right), 2.73-2.76\left(\mathrm{~m}, 1 \mathrm{H}, \mathrm{CH}_{2}-14\right), 3.81$ (s, $3 \mathrm{H}, \mathrm{CH}_{3}$ of $\left.\mathrm{OMe}\right), 3.82\left(\mathrm{~s}, 3 \mathrm{H}, \mathrm{CH}_{3}\right.$ of $\left.\mathrm{OMe}\right), 4.24(\mathrm{q}, 1 \mathrm{H}, J$ $=6.8 \mathrm{~Hz}, \mathrm{CH}-12), 4.45-4.53(\mathrm{~m}, 1 \mathrm{H}, \mathrm{CH}-6), 6.64-6.67(\mathrm{~m}$, $2 \mathrm{H}, \mathrm{CH}-17,21), 6.75$ (d, $1 \mathrm{H}, J=7.9 \mathrm{~Hz}, \mathrm{CH}-20) ;{ }^{13} \mathrm{C}-\mathrm{NMR}$ $\left(\mathrm{CDCl}_{3}, 100 \mathrm{MHz}\right): \delta 20.8(22), 25.2,25.8,33.4,33.4(7,8$, 9, 10, 11), 36.3 (15), 49.0 (14), 49.1 (12), 56.2, 56.3 (24, 26), 58.2 (6), 111.7 (20), 112.2 (21), 120.9 (17), 132.3 (16), 148.0, $149.4(18,19), 156.4(5)$.

$N$-((4-chlorophenyl)(1-cyclohexyl-1H-tetrazol-5yl)methyl)-2-(3,4-dimethoxyphenyl)ethanamine (4f). The general method was followed using homoveratrylamine (5a) (500 
$\mathrm{mg}, 2.76 \mathrm{mmol})$, 4-chlorobenzaldehyde (6e) (388 mg, 2.76 $\mathrm{mmol}$ ), cyclohexylisocyanide (7a) (301 $\mathrm{mg}, 2.76 \mathrm{mmol})$, and azidotrimethylsilane (8) $(318 \mathrm{mg}, 2.76 \mathrm{mmol})$, producing 1145 $\mathrm{mg}(91 \%)$ of $\mathbf{4 f}$ as a white solid; $\mathrm{R}_{f}=0.19$ Hex-AcOEt (3:1 V/V); m.p. 109-110 ${ }^{\circ}$; FT-IR (ATR) $v_{\text {max }} 3301,1514,1471$, 1275, 1260, 768, $750 \mathrm{~cm}^{-1} ;{ }^{1} \mathrm{H}-\mathrm{NMR}\left(\mathrm{CDCl}_{3}, 400 \mathrm{MHz}\right): \delta$ 1.20-1.23 (m, 3H, $\mathrm{CH}_{2}$ of $\left.\mathrm{Cy}\right), 1.48\left(\mathrm{~d}, 1 \mathrm{H}, J=10.5 \mathrm{~Hz}, \mathrm{CH}_{2}\right.$ of $\mathrm{Cy}), 1.58\left(\mathrm{~d}, 1 \mathrm{H}, J=11.0 \mathrm{~Hz}, \mathrm{CH}_{2}\right.$ of $\left.\mathrm{Cy}\right), 1.66\left(\mathrm{~s}, 1 \mathrm{H}, \mathrm{CH}_{2}\right.$ of Cy), 1.81 (t, $4 \mathrm{H}, J=9.5 \mathrm{~Hz}, \mathrm{CH}_{2}$ of $\left.\mathrm{Cy}\right), 2.18(\mathrm{~s}, 1 \mathrm{H}, \mathrm{NH}-$ 13), 2.73-2.82 (m, $\left.4 \mathrm{H}, \mathrm{CH}_{2}-14,15\right), 3.82$ (s, $3 \mathrm{H}, \mathrm{CH}_{3}$ of $\mathrm{OMe}$ ), 3.83 (s, $3 \mathrm{H}, \mathrm{CH}_{3}$ of OMe), 4.21 (tt, $1 \mathrm{H}, J=11.5,3.8 \mathrm{~Hz}, \mathrm{CH}-6$ ), 5.21 (s, 1H, CH-12), 6.67-6.71 (m, 2H, CH-20, 21), 6.77 (d, $1 \mathrm{H}, J=8.1 \mathrm{~Hz}, \mathrm{CH}-17), 7.22(\mathrm{~d}, 2 \mathrm{H}, J=8.1 \mathrm{~Hz}, \mathrm{CH}-23,27)$, $7.29(\mathrm{~d}, 2 \mathrm{H}, J=8.4 \mathrm{~Hz}, \mathrm{CH}-24,26) ;{ }^{13} \mathrm{C}-\mathrm{NMR}\left(\mathrm{CDCl}_{3}, 100\right.$ MHz): $\delta 25.1,25.7,33.0$ (7, 8, 9, 10, 11), 36.2 (15), 49.3 (14), $56.3,56.3(29,31), 57.3(12), 58.4(6), 111.8(17), 112.3,121.0$ $(20,21), 128.9(23,27), 129.6(24,26), 132.2(16), 134.8(25)$ 137.0 (22), 148.1, $149.4(18,19), 154.6(5)$.

2-(3,4-dimethoxyphenyl)-N-((1-(2,6-dimethylphenyl)- $1 \mathrm{H}$ tetrazol-5-yl)(furan-3-yl)methyl)ethanamine (4g). The general method was followed using homoveratrylamine (5a) $(250 \mathrm{mg}$, $1.38 \mathrm{mmol}$ ), 3-furaldehyde (6f) (133 mg, $1.38 \mathrm{mmol}), 2,6$-dimethylphenyl isocyanide (7d) $(181 \mathrm{mg}, 1.38 \mathrm{mmol})$, and azidotrimethylsilane (8) $(159 \mathrm{mg}, 1.38 \mathrm{mmol})$, producing $502 \mathrm{mg}$ $(84 \%)$ of $4 \mathrm{~g}$ as a white solid; $\mathrm{R}_{f}=0.65 \mathrm{Hex}-\operatorname{AcOEt}(3: 1 \mathrm{~V} / \mathrm{V})$; m.p. $63-64^{\circ} \mathrm{C}$; FT-IR (ATR) $v_{\max } 3326,1514,1258,1234$, 1139, $1025 \mathrm{~cm}^{-1}$; ${ }^{1} \mathrm{H}-\mathrm{NMR}\left(\left(\mathrm{CD}_{3}\right)_{2} \mathrm{SO}, 300 \mathrm{MHz}\right): \delta 1.57(\mathrm{~s}$, $3 \mathrm{H}, \mathrm{CH}_{3}$ of $\left.\mathrm{Ph} M e_{2}\right), 1.90$ (s, 3H, $\mathrm{CH}_{3}$ of $\left.\mathrm{Ph} M e_{2}\right), 2.26$ (d, $1 \mathrm{H}$, $J=14.4 \mathrm{~Hz}, \mathrm{NH}-7), 2.55-2.70$ (m, $\left.4 \mathrm{H}, \mathrm{CH}_{2}-8,9\right), 3.76$ (s, $6 \mathrm{H}$, $\left.\mathrm{CH}_{3}-22,24\right), 4.79(\mathrm{CH}-6), 6.41(\mathrm{~d}, 1 \mathrm{H}, J=1.4 \mathrm{~Hz}, \mathrm{CH}-4)$, 6.63-6.68 (m, 2H, CH-14, 15), 6.77 (dd, $1 \mathrm{H}, J=7.9,4.1 \mathrm{~Hz}$, $\mathrm{CH}-11), 7.23-7.29$ (m, 3H, CH-2, 27, 29), 7.40-7.47 (m, 2H, $\mathrm{CH}-5,28) ;{ }^{13} \mathrm{C}-\mathrm{NMR}\left(\left(\mathrm{CD}_{3}\right)_{2} \mathrm{SO}, 75 \mathrm{MHz}\right): \delta 14.9,15,3$ (31, 32), 33.3 (9), 46.3 (8, 6), 53.8, 53.9 (22, 24), 107.7 (4), 110,1 (11), 110.6 (14), 118.7, (15), 126.9, $127.0(27,29), 129.2$ (28), 129.6 (3), 130.4 (25), 133.8, 133.9 (26, 32), 139.1 (2), 141.9 (5), 145.6, $147.0(12,13), 154.6(16)$.

2-(3,4-dimethoxyphenyl)- $N-((1-(2,6-$ dimethylphenyl)- $1 H$ tetrazol-5-yl)(furan-2-yl)methyl)ethanamine (4h). The general method was followed using homoveratrylamine (5a) $(180 \mathrm{mg}$, $0.99 \mathrm{mmol}), 2$-furaldehyde (6g) $(95 \mathrm{mg}, 0.99 \mathrm{mmol}), 2,6$-dimethylphenyl isocyanide (7d) $(130 \mathrm{mg}, 0.99 \mathrm{mmol})$, and azidotrimethylsilane (8) (114 mg, $0.99 \mathrm{mmol})$, producing $379 \mathrm{mg}$ $(88 \%)$ of $\mathbf{4 h}$ as a yellow solid; $\mathrm{R}_{f}=0.68 \mathrm{Hex}-\operatorname{AcOEt}(3: 1 \mathrm{~V} / \mathrm{V})$; m.p. 84-86 ${ }^{\circ}$ C; FT-IR (ATR) $v_{\max } 3326,1514,1257,1233$, $1141,1025 \mathrm{~cm}^{-1}$; ${ }^{1} \mathrm{H}-\mathrm{NMR}\left(\mathrm{CDCl}_{3}, 200 \mathrm{MHz}\right): \delta 1.60(\mathrm{~s}, 3 \mathrm{H}$, $\mathrm{CH}_{3}$ of $\mathrm{PhMe}$ ), 1.91 (s, $3 \mathrm{H}, \mathrm{CH}_{3}$ of $\mathrm{PhMe}$ ), 2.16 (s, $1 \mathrm{H}, \mathrm{NH}-$ 7), 2.76 (dd, $\left.4 \mathrm{H}, J=14.2,5.2, \mathrm{~Hz}, \mathrm{CH}_{2}-8,9\right), 3.84$ (s, 3H, $\mathrm{CH}_{3}$ of $\mathrm{OMe}$ ), 3.86 (s, $3 \mathrm{H}, \mathrm{CH}_{3}$ of $\mathrm{OMe}$ ), 4.83 (s, $\left.1 \mathrm{H}, \mathrm{CH}-6\right), 6.03$ (d, $1 \mathrm{H}, J=3.1 \mathrm{~Hz}, \mathrm{CH}-3$ ), 6.25 (dd, $1 \mathrm{H}, J=3.0,1.8, \mathrm{~Hz}, \mathrm{CH}-$ 4), 6.67-6.68 (m, 2H, CH-11, 14), 6.75-6.79 (m, 1H, CH-15), 7.11-7.21 (m, 2H, CH-27, 29), $7.28(\mathrm{~d}, 1 \mathrm{H}, J=3.5 \mathrm{~Hz}, \mathrm{CH}-5)$, 7.36 (t, $1 \mathrm{H}, J=7.6 \mathrm{~Hz}, \mathrm{CH}-28) ;{ }^{13} \mathrm{C}-\mathrm{NMR}\left(\mathrm{CDCl}_{3}, 50 \mathrm{MHz}\right)$ : $\delta$ 17.1, $17.5(31,32), 35.9(9), 47.8(8), 51.0(6), 56.0,56.1$ (22, 24), 109.0 (4), 110.7 (3), 111.4 (15), 112.0 (11), 120.7 (14), 128.8, 128.9 (27, 29), 131.1 (28), 131.7 (25), 132.0 (10),
135.8, $136.7(26,30), 143.1(5), 147.7,149.1(12,13), 150.2$ (2), $155.2(16)$.

$\mathrm{N}$-(2-(1H-indol-3-yl)ethyl)-1-(1-(4-methoxybenzyl)-1Htetrazol-5-yl)-3-methylbutan-1-amine (4i). The general method was followed using tryptamine (5b) $(500 \mathrm{mg}, 3.12 \mathrm{mmol})$, isovaleraldehyde (6a) (269 mg, $3.12 \mathrm{mmol}$ ), 4-methoxy-benzylisocyanide (7b) (459 mg, $3.12 \mathrm{mmol})$, and azidotrimethylsilane (8) (360 mg, $3.12 \mathrm{mmol})$, producing $1272 \mathrm{mg}(94 \%)$ of $4 \mathbf{i}$ as an amber oil; $\mathrm{R}_{f}=0.42 \mathrm{Hex}-\mathrm{AcOEt}(3: 1 \mathrm{~V} / \mathrm{V})$; FT-IR (ATR) $v_{\max } 3320,3056,1612,1456,1249,1177 \mathrm{~cm}^{-1}$; ${ }^{1} \mathrm{H}-\mathrm{NMR}$ $\left(\mathrm{CDCl}_{3}, 400 \mathrm{MHz}\right): \delta 0.53\left(\mathrm{~d}, 3 \mathrm{H}, J=6.4 \mathrm{~Hz}, \mathrm{CH}_{3}\right.$ of $\left.i-\mathrm{Bu}\right)$, 0.79 (d, $3 \mathrm{H}, J=6.4 \mathrm{~Hz}, \mathrm{CH}_{3}$ of $\left.i-\mathrm{Bu}\right), 1.30$ (tt, $1 \mathrm{H}, J=13.2$, $6.5 \mathrm{~Hz}, \mathrm{CH}-29), 1.39-1.54$ (m, 3H, $\left.\mathrm{CH}_{2}-28, \mathrm{NH}-14\right), 2.61-2.67$ (m, $\left.1 \mathrm{H}, \mathrm{CH}_{2}-16\right), 2.71-2.77$ (m, 1H, $\left.\mathrm{CH}_{2}-16\right), 2.85$ (t, $2 \mathrm{H}, J=$ $\left.6.5 \mathrm{~Hz}, \mathrm{CH}_{2}-15\right), 3.76\left(\mathrm{~s}, 3 \mathrm{H}, \mathrm{CH}_{3}-27\right), 4.18$ (t, $1 \mathrm{H}, J=7.5$ $\mathrm{Hz}, \mathrm{CH}-13), 5.42$ (d, $\left.1 \mathrm{H}, J=15.6 \mathrm{~Hz}, \mathrm{CH}_{2}-6\right), 5.44(\mathrm{~d}, 1 \mathrm{H}$, $\left.J=15.6 \mathrm{~Hz}, \mathrm{CH}_{2}-6\right), 6.81-6.83(\mathrm{~m}, 2 \mathrm{H}, \mathrm{CH}-9,11), 6.95$ (s, 1H, CH-18), 7.05-7.07 (m, 2H, CH-8, 12), 7.13 (t, 1H, J=7.4 $\mathrm{Hz}, \mathrm{CH}-23), 7.21$ (t, 1H, $J=7.4 \mathrm{~Hz}, \mathrm{CH}-24), 7.38(\mathrm{~d}, 1 \mathrm{H}, J=$ $8.0 \mathrm{~Hz}, \mathrm{CH}-22), 7.54(\mathrm{~d}, 1 \mathrm{H}, J=7.8 \mathrm{~Hz}, \mathrm{CH}-25), 8.36(\mathrm{~s}, 1 \mathrm{H}$, $\mathrm{N} H-19) ;{ }^{13} \mathrm{C}-\mathrm{NMR}\left(\mathrm{CDCl}_{3}, 100 \mathrm{MHz}\right): \delta 22.7(30,31), 25.1$ (29), 26.1 (16), 43.5 (28), 48.0 (15), 50.8 (6), 52.4 (13), 55.7 (27), 111.7 (22), 113.9 (21), 114.7 (9, 11), 119.1 (25), 119.7 (23), 122.4 (18), 122.5 (24), 126.6 (7), 127.8 (17), 129.4 (8, 12), 136.8 (20), 156.9 (5), 160.2 (10).

$\mathrm{N}$-(2-(1H-indol-3-yl)ethyl)-1-(1-cyclohexyl-1H-tetrazol5-yl)-3-methylbutan-1-amine (4j). The general method was followed using tryptamine (5b) $(500 \mathrm{mg}, 3.12 \mathrm{mmol})$, isovaleraldehyde (6a) (269 mg, $3.12 \mathrm{mmol}$ ), cyclohexylisocyanide (7a) $(341 \mathrm{mg}, 3.12 \mathrm{mmol})$, and azidotrimethylsilane (8) (360 $\mathrm{mg}, 3.12 \mathrm{mmol})$, producing $1009 \mathrm{mg}(85 \%)$ of $\mathbf{4 j}$ as a white solid; $\mathrm{R}_{f}=0.61 \mathrm{Hex}-\mathrm{AcOEt}(3: 1 \mathrm{~V} / \mathrm{V})$; m.p. $99-101^{\circ} \mathrm{C}$; FT-IR (ATR) $v_{\max } 3310,3157,1619,1454,1119,1101 \mathrm{~cm}^{-1} ;{ }^{1} \mathrm{H}-\mathrm{NMR}$ $\left(\mathrm{CDCl}_{3}, 400 \mathrm{MHz}\right): \delta 0.86\left(\mathrm{~d}, 3 \mathrm{H}, J=6.6 \mathrm{~Hz}, \mathrm{CH}_{3}\right.$ of $\left.i-\mathrm{Bu}\right)$, $0.92\left(\mathrm{~d}, 3 \mathrm{H}, J=6.6 \mathrm{~Hz}, \mathrm{CH}_{3}\right.$ of $\left.i-\mathrm{Bu}\right), 1.28-1.40\left(\mathrm{~m}, 3 \mathrm{H}, \mathrm{CH}_{2}\right.$ of $\mathrm{Cy}, \mathrm{CH}-26), 1.41-1.49\left(\mathrm{~m}, 2 \mathrm{H}, \mathrm{CH}_{2}\right.$ of $\left.\mathrm{Cy}\right), 1.55(\mathrm{~s}, 1 \mathrm{H}$, $\mathrm{NH}-13), 1.62-1.71\left(\mathrm{~m}, 2 \mathrm{H}, \mathrm{CH}_{2}-25\right), 1.92-2.07\left(\mathrm{~m}, 6 \mathrm{H}, \mathrm{CH}_{2}\right.$ of $\mathrm{Cy}), 2.69-2.75\left(\mathrm{~m}, 1 \mathrm{H}, \mathrm{CH}_{2}-14\right), 2.80-2.86\left(\mathrm{~m}, 1 \mathrm{H}, \mathrm{CH}_{2}-14\right)$, 2.88-2.94 (m, 2H, $\left.\mathrm{CH}_{2}-15\right), 4.25$ (t, $1 \mathrm{H}, J=7.4 \mathrm{~Hz}, \mathrm{CH}-12$ ), 4.60 (tt, $1 \mathrm{H}, J=11.4,3.6, \mathrm{~Hz}, \mathrm{CH}-6), 7.00$ (s, 1H, CH-17), 7.12 (t, $1 \mathrm{H}, J=7.4 \mathrm{~Hz}, \mathrm{CH}-22$ ), 7.12 (t, $1 \mathrm{H}, J=7.5 \mathrm{~Hz}, \mathrm{CH}-$ 23 ), 7.38 (d, $1 \mathrm{H}, J=8.1 \mathrm{~Hz}, \mathrm{CH}-21), 7.56(\mathrm{~d}, 1 \mathrm{H}, J=7.9 \mathrm{~Hz}$, $\mathrm{CH}-24), 8.05$ (s, $1 \mathrm{H}, \mathrm{N} H-18) ;{ }^{13} \mathrm{C}-\mathrm{NMR}\left(\mathrm{CDCl}_{3}, 100 \mathrm{MHz}\right): \delta$ 22.9, $23.1(27,28), 24.9$ (9), 25.3 (26), 25.8, 25.9, 32.6, 34.0 (7, 8, 10, 11), 35.7 (15), 43.7 (25), 51.4 (14), 52.7 (12), 111.3 (16), 111.5 (24), 116.5 (21), 120.2, (22) 121.3 (23), 123.2 (17) 127.8 (20), 137.0 (19), 162.7 (5).

$\mathrm{N}$-(2-(1H-indol-3-yl)ethyl)-1-(1-cyclohexyl-1H-tetrazol-5$y$ l)ethanamine (4k). The general method was followed using tryptamine (5b) $(500 \mathrm{mg}, 3.12 \mathrm{mmol})$, acetaldehyde (6d) (137 $\mathrm{mg}, 3.12 \mathrm{mmol})$, cyclohexylisocyanide (7a) $(341 \mathrm{mg}, 3.12$ $\mathrm{mmol}$ ), and azidotrimethylsilane (8) (360 $\mathrm{mg}, 3.12 \mathrm{mmol})$, producing $782 \mathrm{mg}(74 \%)$ of $\mathbf{4 k}$ as a white-yellow solid; $\mathrm{R}_{f}=$ 0.35 Hex-AcOEt $(3: 1 \mathrm{~V} / \mathrm{V})$; m.p. $102-104^{\circ} \mathrm{C}$; FT-IR (ATR) $v_{\max } 3392,3182,1617,1455,1434,1146,1117 \mathrm{~cm}^{-1} ;{ }^{1} \mathrm{H}-\mathrm{NMR}$ $\left(\mathrm{CDCl}_{3}, 400 \mathrm{MHz}\right): \delta 1.28-1.39$ (m, $4 \mathrm{H}, \mathrm{CH}_{2}$ of $\mathrm{Cy}$ ), 1.50 (d, 
$\left.2 \mathrm{H}, J=6.8 \mathrm{~Hz}, \mathrm{CH}_{3}-25\right), 1.77(\mathrm{~s}, 1 \mathrm{H}, \mathrm{NH}-13), 1.90-2.07$ (m, $6 \mathrm{H}, \mathrm{CH}$ of $\mathrm{Cy}), 2.79\left(\mathrm{~m}, 1 \mathrm{H}, \mathrm{CH}_{2}-14\right), 2.88-3.01(\mathrm{~m}, 3 \mathrm{H}$, $\left.\mathrm{CH}_{2} 14, \mathrm{CH}_{2}-15\right), 4.30$ (q, $1 \mathrm{H}, J=6.8 \mathrm{~Hz}, \mathrm{CH}-12$ ), 4.57 (tt, $1 \mathrm{H}$, $J=11.1,3.7 \mathrm{~Hz}, \mathrm{CH}-6), 7.03(\mathrm{~d}, 1 \mathrm{H}, J=2.1 \mathrm{~Hz}, \mathrm{CH}-17), 7.13$ (t, $1 \mathrm{H}, J=7.9 \mathrm{~Hz}, \mathrm{CH}-22), 7.21(\mathrm{t}, 1 \mathrm{H}, J=7.1 \mathrm{~Hz}, \mathrm{CH}-23), 7.38$ (d, $1 \mathrm{H}, J=8.1 \mathrm{~Hz}, \mathrm{CH}-24), 7.58$ (d, $1 \mathrm{H}, J=7.8 \mathrm{~Hz}, \mathrm{CH}-21$ ), 8.11 (s, $1 \mathrm{H}, \mathrm{NH}-18) ;{ }^{13} \mathrm{C}-\mathrm{NMR}\left(\mathrm{CDCl}_{3}, 100 \mathrm{MHz}\right): \delta 21.0(25)$, 25.3 (9), 25.8 (8, 10), 26.2 (15), 33.5 (7, 11), 48.1 (14), 49.3 (12), 58.3 (6), $111.6(24), 113.9$ (16), 119.1 (21), 119.8 (22), 122.3 (17) 122.5 (23), 127.8 (20), 136.8 (19), 156.5 (5).

$\mathrm{N}-($ benzo $[d][1,3]$ dioxol-5-yl(1-(2,6-dimethylphenyl)- $1 \mathrm{H}$ tetrazol-5-yl)methyl)-2-(1H-indol-3-yl)ethanamine (41). The general method was followed using tryptamine (5b) $(350 \mathrm{mg}$, $2.18 \mathrm{mmol}), 1,3$-Benzodioxole-5-carbaldehyde (6h) $(328 \mathrm{mg}$, $2.18 \mathrm{mmol}), 2,6$-dimethylphenyl isocyanide (7d) $(287 \mathrm{mg}, 2.18$ $\mathrm{mmol}$ ), and azidotrimethylsilane (8) $(252 \mathrm{mg}, 2.18 \mathrm{mmol})$, producing $897 \mathrm{mg}(88 \%)$ of $\mathbf{4 I}$ as a yellow solid; $\mathrm{R}_{f}=0.29 \mathrm{Hex}-$ AcOEt $(3: 1 \mathrm{~V} / \mathrm{V})$; m.p. $70-72^{\circ} \mathrm{C}$; FT-IR (ATR) $v_{\max } 3409$, 3311, 1608, 1501, 1483, 1242, $1100 \mathrm{~cm}^{-1}$; ${ }^{1} \mathrm{H}-\mathrm{NMR}\left(\mathrm{CDCl}_{3}\right.$, $300 \mathrm{MHz}$ ): $\delta 1.30$ (d, $3 \mathrm{H}, J=1.8 \mathrm{~Hz}, \mathrm{CH}_{3}$ of $\mathrm{PhMe}$ ), 1.84 (d, $3 \mathrm{H}, J=1.8 \mathrm{~Hz}, \mathrm{CH}_{3}$ of $\left.\mathrm{PhMe} e_{2}\right), 2.23$ (s, $\left.1 \mathrm{H}, \mathrm{NH}-11\right), 2.84-2.96$ (m, 4H, $\mathrm{CH}_{2}-12,13$ ), 4.57 (d, $1 \mathrm{H}, J=2.3 \mathrm{~Hz}, \mathrm{CH}-10$ ), 5.87 (dd, $\left.1 \mathrm{H}, J=2.6,1.7 \mathrm{~Hz}, \mathrm{CH}_{2}-2\right), 6.29$ (dt, $1 H, J=8.0,2.0 \mathrm{~Hz}$, $\mathrm{CH}-9$ ), 6.53 (dd, 1H, J=7.9, $2.5 \mathrm{~Hz}, \mathrm{CH}-8), 6.57$ (t, 1H, $J=$ $1.8 \mathrm{~Hz}, \mathrm{CH}-6), 6.99$ (s, 1H, CH-15), 7.02-7.07 (m, 2H, CH-19, 22), 7.13-7.17 (m, $2 \mathrm{H}, \mathrm{CH}-30,32), 7.30-7.33$ (m, $2 \mathrm{H}, \mathrm{CH}-20$, 21), 7.47 (d, 1H, $J=7.9 \mathrm{~Hz}, \mathrm{CH}-31), 8.31$ (s, $1 \mathrm{H}, \mathrm{NH}-16$ ); ${ }^{13} \mathrm{C}-\mathrm{NMR}\left(\mathrm{CDCl}_{3}, 75 \mathrm{MHz}\right): \delta$ 16.7, $17.1(34,35), 25.5(13)$, 47.4 (12), 57.5 (10), 101.1 (2), 107.7 (6), 107.9 (8), 111.2 (20), 113.0 (14), 118.5 (31), 119.0 (19), 121.3 (9), 121.8 (30), 122.1 (15), 127.2 (18), 128.5 (31), 128.6 (19), 130.8 (21), 131.2 (28), 131.5 (7), 135.2 (17), 136.3, $136.6(29,33), 147.6,147.9$ (4, 5), 156.9 (23).

$N$-((1-(2,6-dimethylphenyl)- $1 \mathrm{H}$-tetrazol-5$y l)($ phenyl)methyl)-2-(1H-indol-3-yl)ethanamine (4m). The general method was followed using tryptamine (5b) $(158 \mathrm{mg}$, $0.99 \mathrm{mmol})$, benzaldehyde (6i) (105 mg, $0.99 \mathrm{mmol}), 2,6$-dimethylphenyl isocyanide (7d) (129 mg, $0.99 \mathrm{mmol})$, and azidotrimethylsilane (8) $(114 \mathrm{mg}, 0.99 \mathrm{mmol})$, producing $325 \mathrm{mg}$ $(78 \%)$ of $\mathbf{4 m}$ as a white solid; $\mathrm{R}_{f}=0.64$ Hex-AcOEt $(3: 1$ V/V); m.p. 91-93 ${ }^{\circ}$ C; FT-IR (ATR) $v_{\max } 3180,3056,1618$, 1456, 1340, $1105 \mathrm{~cm}^{-1}$; ${ }^{1} \mathrm{H}-\mathrm{NMR}\left(\mathrm{CDCl}_{3}, 200 \mathrm{MHz}\right): \delta 1.15$ (s, $3 \mathrm{H}, \mathrm{CH}_{3}$ of $\mathrm{PhMe} e_{2}$ ), 1.84 (s, $3 \mathrm{H}, \mathrm{CH}_{3}$ of $\mathrm{PhMe}$ ), 2.35 (s, 1H, NH-13), 2.79-2.96 (m, 4H, $\left.\mathrm{CH}_{2}-14,15\right), 4.67$ (s, $1 \mathrm{H}, \mathrm{CH}$ 12), 6.92-7.04 (m, 5H, $\mathrm{CH}-17,22,28,29,32), 7.08-7.20$ (m, $5 \mathrm{H}, \mathrm{CH}-8,23,10,30,31), 7.32(\mathrm{dd}, 2 \mathrm{H}, J=7.6,4.9 \mathrm{~Hz}$, $\mathrm{CH}-9,24), 7.46$ (d, $1 \mathrm{H}, J=7.7 \mathrm{~Hz}, \mathrm{CH}-21), 8.37$ (s, $1 \mathrm{H}$, $\mathrm{N} H-18) ;{ }^{13} \mathrm{C}-\mathrm{NMR}\left(\mathrm{CDCl}_{3}, 50 \mathrm{MHz}\right): \delta 16.7,17.4(25,26)$, 25.8 (15), $47.8\left(1 \mathrm{CH}_{2} 14\right), 58.2$ (12), 111.4 (24), 113.2 (16), 118.8 (21), 119.3 (22), 122.0 (3), 122.3 (17), 127.4 (20), $127.8(28,32), 128.7(30), 128.7(9), 128.9(29,31), 131.1$ (9), 131.6 (6), 135.3 (27), 136.5 (19), 137.07, 137.5 (7, 11), $157.1(5)$.

$N$-((4-chlorophenyl)(1-(4-methoxybenzyl)-1H-tetrazol5-yl)methyl)-2-(1H-indol-3-yl)ethanamine (4n). The general method was followed using tryptamine (5b) $(500 \mathrm{mg}, 3.12$ mmol), 4-chlorobenzaldehyde (6e) (439 mg, $3.12 \mathrm{mmol}$ ), 4methoxy-benzylisocyanide (7b) $(459 \mathrm{mg}, 3.12 \mathrm{mmol})$, and azidotrimethylsilane (8) (360 mg, $3.12 \mathrm{mmol}$ ), producing $1151 \mathrm{mg}$ $(78 \%)$ of $4 \mathbf{n}$ as an amber solid; $\mathrm{R}_{f}=0.47 \mathrm{Hex}-\mathrm{AcOEt}(3: 1 \mathrm{~V} / \mathrm{V})$; m.p. 66-68 ${ }^{\circ}$ C; FT-IR (ATR) $v_{\max } 3405,3309,1612,1513$, 1455, $1248 \mathrm{~cm}^{-1}$; ${ }^{1} \mathrm{H}-\mathrm{NMR}\left(\mathrm{CDCl}_{3}, 400 \mathrm{MHz}\right): \delta 2.07(\mathrm{~s}, 1 \mathrm{H}$, $\mathrm{NH}-14), 2.78-2.89$ (m, 2H, $\left.\mathrm{CH}_{2}-16\right), 2.93$ (t, $2 \mathrm{H}, J=6.4 \mathrm{~Hz}$, $\left.\mathrm{CH}_{2}-15\right), 3.75$ (s, $\left.3 \mathrm{H}, \mathrm{CH}_{3}-27\right), 5.05(\mathrm{~s}, 1 \mathrm{H}, \mathrm{CH}-13), 5.26(\mathrm{~s}$, $\left.1 \mathrm{H}, \mathrm{CH}_{2}-6\right), 6.73(\mathrm{~d}, 2 \mathrm{H}, J=7.3 \mathrm{~Hz}, \mathrm{CH}-9,11), 6.82(\mathrm{~d}, 2 \mathrm{H}$, $J=7.6 \mathrm{~Hz}, \mathrm{CH}-8,12), 6.96$ (s, 1H, CH-18), 7.03 (d, 2H, $J=$ $7.6 \mathrm{~Hz}, \mathrm{CH}-29,33), 7.12$ (t, $1 \mathrm{H}, J=7.5 \mathrm{~Hz}, \mathrm{CH}-23$ ), 7.16-7.24 (m, 3H, CH-30, 32, 24), 7.36 (d, 1H, $J=81 \mathrm{~Hz}, \mathrm{CH}-25), 7.52$ (d, $1 \mathrm{H}, J=7.9 \mathrm{~Hz}, \mathrm{CH}-22), 8.58$ (s, $1 \mathrm{H}, N H-19) ;{ }^{13} \mathrm{C}-\mathrm{NMR}$ $\left(\mathrm{CDCl}_{3}, 100 \mathrm{MHz}\right): \delta 25.1$ (16), 48.0 (15), 51.0 (6), 55.8 (27), 55.3, (13), 111.9 (25), 113.5 (17), 114.7 (9,11), 119.1 (22), 119.7 (23), 122.5 (24), 122.8 (18), 125.6, (21) 127.7 (7), 129.2 $(29,33) 129.3(8,12), 129.48(30,32), 134.6(31), 136.4(28)$, 136.9 (20), 155.9 (5), 160.2 (10).

$\mathrm{N}$-(2-(1H-indol-3-yl)ethyl)-1-(1-(2,6-dimethylphenyl)-1Htetrazol-5-yl)-2-phenylethanamine (4o). The general method was followed using tryptamine (5b) $(183 \mathrm{mg}, 1.14 \mathrm{mmol})$, 2-phenylacetaldehyde (6j) (137 mg, $1.14 \mathrm{mmol}), 2,6$-dimethylphenyl isocyanide (7d) $(150 \mathrm{mg}, 1.14 \mathrm{mmol})$, and azidotrimethylsolane (8) (132 mg, $1.14 \mathrm{mmol}$ ), producing $349 \mathrm{mg}$ (70\%) of 40 as a yellow solid; $\mathrm{R}_{f}=0.50 \mathrm{Hex}-\mathrm{AcOEt}(3: 1 \mathrm{~V} / \mathrm{V}) ; \mathrm{m} . \mathrm{p}$. $56-57^{\circ} \mathrm{C}$; FT-IR (ATR) $v_{\text {max }} 3412,3321,1717,1471,1455$, $1092 \mathrm{~cm}^{-1} ;{ }^{1} \mathrm{H}-\mathrm{NMR}\left(\mathrm{CDCl}_{3}, 300 \mathrm{MHz}\right): \delta 1.36\left(\mathrm{~s}, 3 \mathrm{H}, \mathrm{CH}_{3}\right.$ of $\left.\mathrm{PhCH}_{3}\right), 1.84$ (s, 3H, $\mathrm{CH}_{3}$ of $\left.\mathrm{PhCH}_{3}\right), 2.73-2.82\left(\mathrm{~m}, 2 \mathrm{H}, \mathrm{CH}_{2}-\right.$ 15), 2.82-2.98 (m, 2H, $\mathrm{CH}_{2}-14$ ), 3.15 (ddd, $2 \mathrm{H}, J=19.8,13.3$, 7.2, $\mathrm{Hz}, \mathrm{CH}_{2}-27$ ), 3.79 (dd, $1 \mathrm{H}, J=7.7,6.6 \mathrm{~Hz}, \mathrm{CH}-12$ ), 6.79 (d, $1 \mathrm{H}, J=2.3 \mathrm{~Hz}, \mathrm{CH}-17), 6.92-6.97$ (m, 2H, C-17), 7.04-7.14 $\left(\mathrm{m}, 5 \mathrm{H}, \mathrm{C} H_{\text {arom }}\right), 7.14-7.22\left(\mathrm{~m}, 2 \mathrm{H}, \mathrm{CH}_{\text {arom }}\right), 7.32(\mathrm{t}, 2 \mathrm{H}, J=$ $\left.7.9 \mathrm{~Hz}, \mathrm{CH}_{\text {arom }}\right), 7.46\left(\mathrm{~d}, 1 \mathrm{H}, J=7.9 \mathrm{~Hz}, \mathrm{CH}_{\text {arom }}\right), 8.14(\mathrm{~s}, 1 \mathrm{H}$, $\mathrm{NH}-18) ;{ }^{13} \mathrm{C}-\mathrm{NMR}\left(\mathrm{CDCl}_{3}, 75 \mathrm{MHz}\right): \delta 16.7,17.5(25,26)$, 25.9 (15); 39.5 (27); 47.0 (14); 54.8 (12), 111.4, (24); 113.5 (16); 118.8 (21); 119.4, 122.1 (22, 23); 122.2 (17); 126.9, (31); 127.5 (16); 128.5, 128.6, 128.7, 128.8, 128.9, 129.6, $130.9(8$, $9,10,29,30,32,33) ; 132.0$ (6) 136.0 (19); 136.5,136.6 (7, 11), 137.3 (28), $157.3(5)$.

$\mathrm{N}$-((4-chlorophenyl)(1-(2,6-dimethylphenyl)-1H-tetrazol5-yl)methyl)-2-(4-methoxyphenyl)ethanamine (4p): The general method was followed using 4-methoxyphenethylamine (5c) $(500 \mathrm{mg}, 3.31 \mathrm{mmol})$, 4-chlorobenzaldehyde (6e) (465 $\mathrm{mg}, 3.31 \mathrm{mmol}), 2,6$-dimethylphenyl isocyanide (7d) $(434 \mathrm{mg}$, $3.31 \mathrm{mmol})$, and azidotrimethylsilane (8) $(381 \mathrm{mg}, 3.31 \mathrm{mmol})$, producing $1422 \mathrm{mg}(96 \%)$ of $\mathbf{4 p}$ as a white solid; $\mathrm{R}_{f}=0.46$ Hex-AcOEt (3:1 V/V); m.p. $118-119^{\circ} \mathrm{C}$; FT-IR (ATR) $v_{\max }$ 3293, 1513, 1275, 1260, 1089, $1024 \mathrm{~cm}^{-1}$; ${ }^{1} \mathrm{H}-\mathrm{NMR}\left(\mathrm{CDCl}_{3}\right.$, $300 \mathrm{MHz}$ ): $\delta 1.27$ (s, $3 \mathrm{H}, \mathrm{CH}_{3}$ of $\left.\mathrm{PhMe} e_{2}\right), 1.90\left(, 3 \mathrm{H}, \mathrm{CH}_{3}\right.$ of $\mathrm{Ph} \mathrm{Me}_{2}$ ), 2.23 (s, 1H, $\left.\mathrm{NH}-13\right), 2.67-2.81$ (m, 4H, $\left.\mathrm{CH}_{2}-14,15\right)$, 3.77 (s, $\left.3 \mathrm{H}, \mathrm{CH}_{3}-31\right), 4.63$ (s, $\left.1 \mathrm{H}, \mathrm{CH}-12\right), 6.78$ (d, $2 \mathrm{H}, J=8.2$ $\mathrm{Hz}, \mathrm{CH}-17,21), 6.93$ (d, 2H, J=8.3 Hz, CH-25, 29), 7.02-7.08 (m, 3H, CH-8, 26, 28), 7.16-7.23 (m, 3H, CH-10, 17, 21), 7.37 (t, $1 \mathrm{H}, J=7.6 \mathrm{~Hz}, \mathrm{CH}-9) ;{ }^{13} \mathrm{C}-\mathrm{NMR}\left(\mathrm{CDCl}_{3}, 75 \mathrm{MHz}\right): \delta 16.7$, 17.3 (22, 23), 35.3 (31), 48.9 (15), 55.2 (14), 57.2 (12), 113.9 $(17,21), 128.7,128.8(8,10), 128.9(17,21), 129.1(25,29)$, 
$129.5(26,28), 131.0$ (9), 131.3 (6), 131.5 (16), 134.4 (27), 135.3, 136.0 (7, 11), 136.6 (24), 156.5 (5), 158.28 (19).

1-(1-(2,6-dimethylphenyl)-1H-tetrazol-5-yl)-N-(3-methoxyphenethyl)-2-methylpropan-1-amine (4q): The general method was followed using 3-methoxyphenethylamine (5d) (350 $\mathrm{mg}, 2.31 \mathrm{mmol})$, isobutyraldehyde (6b) $(167 \mathrm{mg}, 2.31$ mmol), 2,6-dimethylphenyl isocyanide (7d) $(253 \mathrm{mg}, 2.31$ $\mathrm{mmol}$ ), and azidotrimethylsilane (8) (304 $\mathrm{mg}, 2.31 \mathrm{mmol})$, producing $782 \mathrm{mg}(89 \%)$ of $\mathbf{4 q}$ as a yellow oil; $\mathrm{R}_{f}=0.50 \mathrm{Hex}-$ AcOEt (3:1 V/V); FT-IR (ATR) $v_{\text {max }} 3339,1466,1259,1151$, 1099, $1039 \mathrm{~cm}^{-1} ;{ }^{1} \mathrm{H}-\mathrm{NMR}\left(\mathrm{CDCl}_{3}, 300 \mathrm{MHz}\right): \delta 0.88(\mathrm{dd}, 3 \mathrm{H}$, $\left.J=20.8,6.7 \mathrm{~Hz}, \mathrm{CH}_{3}-25,28\right), 1.63$ (s, $\left.1 \mathrm{H}, \mathrm{N} H-13\right), 1.91,1.93$ (s, 3H, $\mathrm{CH}_{3}$ of $\left.\mathrm{PhMe} e_{2}\right), 2.01-2.11$ (m, 1H, CH-24), 2.64-2.74 (m, 3H, $\left.\mathrm{CH}_{2}-14,15\right), 2.82-2.89\left(\mathrm{~m}, 1 \mathrm{H}, \mathrm{CH}_{2}-14\right), 3.39(\mathrm{~d}, 1 \mathrm{H}$, $J=6.3 \mathrm{~Hz}, \mathrm{CH}-12), 3.77$ (s, 3H, $\left.\mathrm{CH}_{3}-27\right), 6.70-6.74$ (m, 3H, $\mathrm{CH}-17,19,21), 7.14-7.22$ (m, 3H, CH-8, 10, 20), 7.37 (t, 1H, $J=7.6 \mathrm{~Hz}, \mathrm{CH}-9) ;{ }^{13} \mathrm{C}-\mathrm{NMR}\left(\mathrm{CDCl}_{3}, 75 \mathrm{MHz}\right): \delta 17.0(25)$, 17.2, 17.3 (22, 23), 19.7 (28), 30.2 (24), 36.5 (14), 47.7 (15), 54.9 (27), 57.9 (12), 111.4, 114.2, $120.8(17,19,21), 128.7$ (8, 10), 129.1 (20), $130.6(9), 131.8(6), 135.4,135.6(7,11), 141.0$ (16), 156.9 (18), 159.5 (5).

$\mathrm{N}-((1-($ tert-butyl)-1H-tetrazol-5-yl)methyl)-2-(3,4-dimetho xyphenyl)ethanamine (4r): The general method was followed using homoveratrylamine (5a) $(300 \mathrm{mg}, 1.66 \mathrm{mmol})$, paraformaldehyde (6k) $(50 \mathrm{mg}, 1.66 \mathrm{mmol}), t$-butyl isocyanide (7c) $(138 \mathrm{mg}, 1.66 \mathrm{mmol})$, and azidotrimethylsilane (8) $(191 \mathrm{mg}$, $1.66 \mathrm{mmol})$, was stirred overnight under inert atmosphere $\left(\mathrm{N}_{2}\right)$ at room temperature, producing $423 \mathrm{mg}(80 \%)$ of $4 \mathbf{r}$ as a yellow oil; $\mathrm{R}_{f}=0.61 \mathrm{Hex}-\mathrm{AcOEt}(3: 1 \mathrm{~V} / \mathrm{V})$; FT-IR (ATR) $v_{\text {max }} 3322$, 1514, 1463, 1260, $1025 \mathrm{~cm}^{-1}$; ${ }^{1} \mathrm{H}-\mathrm{NMR}\left(\mathrm{CDCl}_{3}, 300 \mathrm{MHz}\right): \delta$ 1.17 (m, 1H, NH-9), 1.70 (s, 9H, $\left.\mathrm{CH}_{3}-7,18,19\right), 2.76-2.80$ (m, $2 \mathrm{H}, \mathrm{CH}_{2}-11$ ), 2.89-2.95 (m, 2H, $\left.\mathrm{CH}_{2}-10\right), 3.86$ (t, $6 \mathrm{H}, J=4.8$ $\left.\mathrm{Hz}, \mathrm{CH}_{3}-21,23\right), 4.15$ (d, 2H, J=4.5 Hz, $\left.\mathrm{CH}_{2}-8\right), 6.71-6.75(\mathrm{~m}$, $2 \mathrm{H}, \mathrm{CH}-13,17), 6.77-6.81(\mathrm{~m}, 1 \mathrm{H}, \mathrm{CH}-16) ;{ }^{13} \mathrm{C}-\mathrm{NMR}\left(\mathrm{CDCl}_{3}\right.$, $75 \mathrm{MHz}): \delta 29.2(7,18,19), 35.5$ (11), $44.0(8), 50.4(10), 55.7$, $55.7(21,23), 61.1(6), 111.4,111.9(13,16), 120.4(17), 131.9$ (12), 147.4, 148,8 (14, 15), 152,7 (5).

\section{Acknowledgements}

Financial support from CONACYT (Project 166747 CB2011-01) and post-doctoral (Dr. Alejandro Islas Jácome (CVU 176745, 290662_UG, 290735_UG) and doctoral (Luis Edilberto Cárdenas Galindo, CVU 225689, 209242) and (Carlos Jesús Cortes García, CVU 230777, 209054) fellowships are gratefully acknowledged.

\section{References}

1. García, G.; Rodríguez-Puyol, M.; Alajarín, R.; Serrano, I.; Sánchez-Alonso, P.; Griera, M.; Vaquero, J.; Rodríguez-Puyol, D.; Alvarez-Builla, J.; Díez-Marques, M. J. Med. Chem. 2009, 52, 7220-7227.
2. Herr, R. J. Bioorg. Med. Chem. 2002, 10, 3379-3393.

3. a) Chu, S. S. Drugs Future 1985, 10, 632-635; b) Cannon, J. R.; Eacho, P. I. Biochem. J. 1991, 280, 387-391.

4. Davulcu, A.; Mc Leod, D.; Li, J.; Katipally, K.; Littke, A.; Doubleday, W.; Xu, Z.; McConlogue, C.; Lai, C.; Gleeson, M.; Schwinden, M.; Parsons, R. J. Org. Chem. 2009, 74, 40684079.

5. Koldovskii, G. I.; Kharbash, R. B. Russ. J. Org. Chem. 2003, 39, 453-470.

6. a) For review see: Brase, S.; Gil, C.; Knepper, K.; Zimmermann, V. Angew. Chem. Int. Ed. 2005, 44, 5188-5240; b) Amantini, D.; Beleggia, R.; Fringelli, F.; Pizzo, F.; Vaccaro, F. J. Org. Chem. 2004, 69, 2896-2898; c) Demko, Z. P.; Sharpless, K. B. J. Org. Chem. 2001, 66, 7945-7950; d) Himo, F.; Demko, Z. P.; Noodleman, L.; Sharpless, K. B. J. Am. Chem. Soc. 2003, 125, 9983-9987; e) Alterman, M.; Hallberg, A. J. Org. Chem. 2000, 65, 7984-7989. f) Keith, J. M. J. Org. Chem. 2006, 71, 9540-9543; g) Demko, Z. P.; Sharpless, K. B. Org. Lett. 2002, 4, 2525-2527. h) Athanassopoulos, C. M.; Garnelis, T.; Vahliotis, D.; Papaioannou, D. Org. Lett. 2005, 7, 561-564; i) Kamijo, S.; Jin, T.; Yamamoto, Y. J. Org. Chem. 2002, 67, 7413-7417; j) Shie, J.; Fang, J. J. Org. Chem. 2007, 72, 3141-3144.

7. Aldhoun, M.; Massi, A.; Dondoni, A. J. Org. Chem. 2008, 73, 9565-9575.

8. a) Hajra, S.; Sinha, D.; Bhowmick, M. J. Org. Chem. 2007, 72, 1852-1855; b) Borah, P.; Naidu, P. S.; Buhyan, P. J. Tetrahedron Lett. 2012, 53, 5034-5037; c) El Kaim, L.; Grimaud, L.; Patil, P. Org. Lett. 2011, 13, 1261-1263.

9. Nixey, T.; Hulme, C. Tetrahedron Lett. 2002, 43, 6833-6835.

10. Kazemizadeh, A. R.; Hajaliakbari, N.; Hajian, R.; Shajari, N.; Ramazani, A. Helv. Chim. Acta 2012, 95, 594-597.

11. a) For Review see: El Kaim, L.; Grimaud, L. Tetrahedron 2009, 65, 2153-2171; a) Nixey, T.; Kelly, M.; Semin, D.; Hulme, C. Tetrahedron Lett. 2002, 43, 3681-3684; b) El Kaim, L.; Grimaud, L.; Purumandla, S. R. Synlett. 2012, 23, 295-297; c) Mayer, J.; Umkehrer, M.; Kalinski, C.; Ross, G.; Kolb, J.; Burdack, C.; Hiller, W. Tetrahedron Lett. 2005, 46, 7393-7396; (d) Dömling, A.; Beck, B.; Magnin-Lachaux, M. Tetrahedron Lett. 2006, 47, 4289-4291.

12. Achatz, S.; Dömling, A. Bioorg. Med. Chem. Lett. 2006, 16, 63606362.

13. Marcos, C. F.; Marcaccini, S.; Menchi, G.; Pepino, R.; Torroba, T. Tetrahedron Lett. 2008, 49, 149-152.

14. a) Gunawan, S.; Nichol, G.; Hulme, C. Tetrahedron Lett. 2012, 53, 1664-1667; b) Gunawan, S.; Ayaz, M.; Moliner, F. D.; Frett, B.; Kaiser, C.; Patrick, N.; Xu, X.; Hulme, C. Tetrahedron Lett. 2012, 68, 5606-5611; c) Gunawan, S.; Keck, K.; Laetsch, A.; Hulme, C. Mol. Divers. 2012, 16, 601-606.

15. a) Dömling, A.; Ugi, I. Angew. Chem. Int. Ed. 2000, 39, 31683210; b) Zhu, J. Eur. J. Org. Chem. 2003, 1133-1144; (c) Ramón, D, J.; Yus, M. Angew. Chem. Int. Ed. 2005, 44, 1602-1634; d) Pan, S. C.; List, B. Angew. Chem. Int. Ed. 2008, 47, 3622-3625.

16. Umkehrer, M.; Kolb, J.; Burdack, C.; Ross, G.; Hiller, W. Tetrahedron Lett. 2004, 45, 6421-6424.

17. Nixey, T.; Kelly, M.; Hulme, C. Tetrahedron Lett. 2000, 41, 87298733 .

18. Kalinski, C.; Umkehrer, M.; Gonnard, S.; Jager, N.; Ross, G.; Hiller, W. Tetrahedron Lett. 2006, 47, 2041-2044.

19. Bienaymé, H.; Bouzid, K. Tetrahedron Lett. 1998, 39, $2735-$ 2738.

20. a) Schaffer, E, S.; Höfner, G.; Wanner, K. T. Bioorg. Med. Chem. 2011, 19, 6492-6504; b) Gunawan, S.; Petit, J.; Hulme, C. Comb. Sci. 2012, 14, 160-163; c) Ref. 13.

21. See refs. $8 a, 10,11 a$ and 12 for 1,5 dipolar electrocyclization. 